\title{
A transmission renewal planning index considering transmission loss and voltage magnitude in $\mathrm{N}-1$ contingency
}

\author{
Yu Takamizawa, Fumihiro Nakatani ${ }^{*}$, Shinichi Iwamoto \\ Waseda University, 3-4-1 Ohkubo Shinjuku-ku, Tokyo 169-8555, Japan
}

\begin{abstract}
With the high economic growth era until 1990s, the power demand increased sharply year by year. Therefore, the electric power utilities installed many electric power facilities along with the predicted demand. However, in recent years, fewer apparatuses have been installed because electric power demand growth has started saturated due to low economic growth. Then, for smart electric power facilities planning, it is necessary to form a rational renewal planning from various aspects, which does not consider only facility ages. In this paper, we first propose a new smart transmission renewal planning index using active power loss and reactive power loss sensitivities. And then, we propose to evaluate the decrease of active power flow loss, and the voltage improvement due to the decrease of reactive power flow loss in the N-1 contingency, which are achieved by line renewals. Finally we carry out simulations for the IEEJ EAST 10-machine model system and determine the transmission planning priority to confirm the validity of the proposed method.
\end{abstract}

Keywords: Power system, system planning, transmission system renewal, transmission line, line flow sensitivity, loss sensitivity

\section{Introduction}

Japanese electric power utilities installed many electric power facilities along with the predicted demand during the period of high economic growth extending until the 1990s. In conventional electric power facility planning, the utilities constructed new transmission lines and substations, what is called "expansion work," to match the increasing demand.

In this situation, the aged facilities were renewed necessarily and seldom used over a lifetime [1].

However, in recent years, it has been assumed that the expansion work has transitioned at a low level over the long-term because electric power demand growth has been saturated due to low economic growth. Also, a large amount of the facilities installed during the high economic growth era will gradually reach 40 years of use after about the year 2010. Though there was a time when the renewal of facilities was expected by the expansion work so far, the idea of using the existing facilities for a longer term will be stronger from now on. Due to this tendency, the subject of electric power facility planning has changed from the past expansion work to improvement work. Therefore, a full-fledged renewal time of electric power facilities will be coming soon.

In smart electric power facilities planning, improvement work causes some restrictions to construction which become big problems. To solve these problems, it is necessary to plan the electric power facility renewal planning from "various aspects."

Therefore, in this paper we focus on the transmission renewals and propose a new smart transmission renewal planning index. First we develop the idea of an NICF (network impedance contribution factor) that is the line flow sensitivity for the impedance change of each line. The second is LLS (line loss sensitivity) which represents the line loss sensitivity for the same change, and the third is SLS (system loss sensitivity) which represents the system loss sensitivity for the same change. The last is ESLC

\footnotetext{
* Manuscript received May 16, 2013; revised July 29, 2013.

Corresponding author. E-mail address: iwamoto.lab.pwrs@gmail.com.
} 
(estimated system loss change).

Furthermore, we also propose a new transmission renewal planning prioritizing method using the ESLC considering both the decrease of active power flow loss and the voltage quality improvement due to the decrease of reactive power flow loss in an N-1 contingency. Our proposed method aims to maximally decrease transmission loss and partly improve bus voltage qualities by choosing the transmission lines that have a larger amount of active power flow loss decrease achieved by their renewals.

We carried out simulations to confirm the validity of the above-mentioned proposed method by using the IEEJ EAST 10 machine 47 bus system model [2] and determined the transmission renewal planning priority.

\section{Power Flow Calculation [3]}

As the proposed index is based on the AC power flow calculation, we explain it in this chapter.

\subsection{Bus power}

Defining the bus voltage, $V_{i}$, at the bus $i$ and the bus admittance matrix, $Y_{i j}$, as:

$$
\begin{aligned}
& V_{i}=e_{i}+j f_{i} \\
& Y_{i j}=G_{i j}+j B_{i j}
\end{aligned}
$$

where $e_{i}$ is real part of voltage at bus $i, f_{i}$ is imaginary part of voltage at bus $i, G_{i j}$ is conductance, and $B_{i j}$ is susceptance.

We can express the bus power, $S_{i}$, in the following equation.

$$
S_{i}=\sum_{j=1}^{n}\left(e_{i}+j f_{i}\right)\left(e_{j}+j f_{j}\right)\left(G_{i j}-j B_{i j}\right)=P_{i}+j Q_{i}
$$

where $n$ is the number of buses, $P_{i}$ is bus active power, $Q_{i}$ is conductance, and $B_{i j}$ is bus reactive power.

\subsection{Line flow}

Defining the line conductance as $g_{i j}$ and the line susceptance as $b_{i j}$, we can express the line admittance by the following equation.

$$
y_{i j}=g_{i j}+j b_{i j}
$$

Furthermore we can also express the line flow, $S L_{i j}$, by the following equation.

$$
S L_{i j}=\left(e_{i}+j f_{i}\right)\left\{e_{i}-e_{j}-j\left(f_{i}-f_{j}\right)\right\}\left(g_{i j}-j b_{i j}\right)-j\left(e_{i}^{2}+f_{i}^{2}\right) \frac{y_{i j}^{\prime}}{2}=P L_{i j}+j Q L_{i j}
$$

where $y_{i j}^{\prime} / 2$ is line-charging capacitance, $P L_{i j}$ is active power flow, and $Q L_{i j}$ is reactive power flow.

\section{Proposed Method}

\subsection{NICF}

The line flow sensitivity NICF is calculated by solving the following three equations simultaneously, which are derived from the above power flow equations.

$$
\begin{aligned}
& {\left[\begin{array}{l}
\Delta \mathbf{P L} \\
\Delta \mathbf{Q L}
\end{array}\right]=\left[\begin{array}{l}
\mathbf{J}_{\mathrm{LV}}
\end{array}\right]\left[\begin{array}{l}
\Delta \mathbf{e} \\
\mathbf{\Delta f}
\end{array}\right]} \\
& {\left[\begin{array}{l}
\boldsymbol{\Delta} \mathbf{P} \\
\boldsymbol{\Delta} \mathbf{Q} \\
\boldsymbol{\Delta}|\mathbf{V}|^{2}
\end{array}\right]=\left[\begin{array}{l}
\mathbf{J}_{\mathrm{SV}}
\end{array}\right]\left[\begin{array}{l}
\Delta \mathbf{e} \\
\Delta \mathbf{f}
\end{array}\right]}
\end{aligned}
$$




$$
\left[\begin{array}{l}
\Delta \mathbf{P} \\
\Delta \mathbf{Q} \\
\Delta|\mathbf{V}|^{2}
\end{array}\right]=\left[\begin{array}{l}
\mathbf{J}_{\mathrm{Sy}}
\end{array}\right]\left[\begin{array}{l}
\Delta \mathbf{g} \\
\Delta \mathbf{b}
\end{array}\right]
$$

where the factors of the $J$ matrices are derivatives of the left vectors with respect to the right ones. The factors of the $\Delta|\mathbf{V}|^{2}$ columns of $\mathbf{J}_{\mathbf{S y}}$ are $\mathbf{0}$ in Eq.(8).

Simultaneously solving (6), (7), and (8) gives

$$
\left[\begin{array}{c}
\Delta \mathbf{P L} \\
\Delta \mathbf{Q L}
\end{array}\right]=\left[\mathbf{J}_{\mathrm{LV}}\right]\left[\mathbf{J}_{\mathrm{SV}}\right]^{-1}\left[\begin{array}{l}
\mathbf{J}_{\mathrm{Sy}}
\end{array}\right]\left[\begin{array}{c}
\Delta \mathbf{g} \\
\Delta \mathbf{b}
\end{array}\right]=\left[\begin{array}{l}
\mathbf{N I C F}
\end{array}\right]\left[\begin{array}{c}
\Delta \mathbf{g} \\
\Delta \mathbf{b}
\end{array}\right]
$$

The product of the three matrices above is defined as the NICF, which is a square matrix.

\section{2. $L L S$}

There are two kinds of $\Delta \mathbf{P L}$ and $\Delta \mathbf{Q L}$, whose directions are bus $i \rightarrow$ bus $j$ and bus $j \rightarrow$ bus $i$. Therefore, two NICFs, which are $\mathbf{N I C F}_{i j}$ and $\mathbf{N I C F}_{j i}$, are derived as the line flow sensitivity. In this paper, we derived the LLS by adding up these NICFs. When we define the active power loss change of each line as $\Delta$ Ploss and the reactive one as $\Delta$ Qloss, the LLS can be expressed by the following equations:

$$
\begin{aligned}
{\left[\begin{array}{c}
\Delta \mathbf{P l o s s} \\
\Delta \mathbf{Q l o s s}
\end{array}\right] } & =\left[\begin{array}{c}
\Delta \mathbf{P L}_{i j} \\
\Delta \mathbf{Q L}_{i j}
\end{array}\right]+\left[\begin{array}{l}
\Delta \mathbf{P L}_{j i} \\
\Delta \mathbf{Q L}_{j i}
\end{array}\right]=\left[\mathbf{N I C F}_{i j}\right]\left[\begin{array}{l}
\Delta \mathbf{g} \\
\mathbf{b}
\end{array}\right]+\left[\begin{array}{c}
\mathbf{N I C F}_{j i}
\end{array}\right]\left[\begin{array}{c}
\Delta \mathbf{g} \\
\Delta \mathbf{b}
\end{array}\right] \\
& =\left\{\left[\mathbf{N I C F}_{i j}\right]+\left[\mathbf{N I C F}_{j i}\right]\right\}\left[\begin{array}{l}
\Delta \mathbf{g} \\
\Delta \mathbf{b}
\end{array}\right]=[\mathbf{L L S}]\left[\begin{array}{c}
\Delta \mathbf{g} \\
\Delta \mathbf{b}
\end{array}\right]
\end{aligned}
$$

where the dimension of the LLS is same as that of the NICF.

\subsection{Equation transformations for prioritizing}

The LLS is expressed as follows:

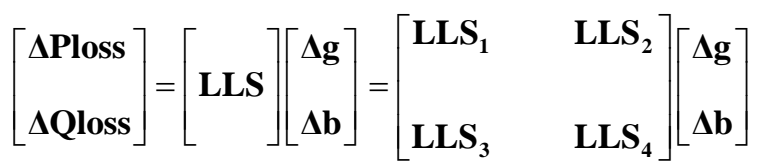

3.4. SLS

The SLS which is system loss sensitivity for the impedance change of each line is derived by adding up the row factors of LLS.

$$
\left[\begin{array}{c}
\sum \Delta \text { Ploss }_{i} \\
\sum \Delta \text { loss }_{i}
\end{array}\right]=\left[\begin{array}{ll}
\mathbf{S L S}_{1} & \mathbf{S L S}_{2} \\
\mathbf{S L S}_{3} & \mathbf{S L S}_{4}
\end{array}\right]\left[\begin{array}{c}
\Delta \mathbf{g} \\
\mathbf{\Delta b}
\end{array}\right]
$$

where $\sum \Delta$ Ploss $_{i}$ is active power loss change of the whole system, $\sum \Delta$ Qloss $_{i}$ is reactive power loss change of the whole system, $\mathbf{S L S}_{n},_{1 i}=\sum_{k=1}^{\text {Nline }} \mathbf{L L S}_{n},{ }_{k i} ; n$ is subscript of SLS $(1 \leq n \leq 4), k$ is number of line, and $i$ is number of row.

\subsection{ESLC}

There are two sensitivities of $\Delta$ Ploss, which are $\mathbf{S L S}_{\mathbf{1}}$ for $\Delta \mathbf{g}$ and $\mathbf{S L S}_{2}$ for $\Delta \mathbf{b}$. In the same way, there are two sensitivities of $\Delta$ Qloss, which are $\mathbf{S L S}_{3}$ for $\Delta \mathbf{g}$ and $\mathbf{S L S}_{4}$ for $\Delta \mathbf{b}$. 
We combined these two sensitivities through our proposed method which made a ranking for $\Delta$ Ploss and $\Delta$ Qloss, respectively. Concretely, we converted $\mathbf{S L S}_{1}$ and $\mathbf{S L S}_{3}$ into the sensitivities for $\Delta \mathbf{b}$, and added them to $\mathbf{S L S}_{2}$ and $\mathbf{S L S}_{4}$ individually. Defining resistance change rate of line $i$ is $\alpha_{i}[\%]$ and reactance one is $\beta_{i}[\%]$, we can show the SLS as follows by multiplying $\gamma_{i}$, which is the ratio of the line impedance, to both $\mathbf{S L S}_{\mathbf{1}}$ and $\mathbf{S L S}_{\mathbf{3}}$, and adding them to $\mathbf{S L S}_{2}$ and $\mathbf{S L S} \mathbf{S}_{4}$ respectively.

$$
\left[\begin{array}{ll}
\mathbf{S L S}_{1} & \mathbf{S L S}_{2} \\
\mathbf{S L S}_{3} & \mathbf{S L S}_{4}
\end{array}\right]\left[\begin{array}{c}
\Delta \mathbf{g} \\
\Delta \mathbf{b}
\end{array}\right]=\left[\begin{array}{l}
\mathbf{S L S}_{1}{ }^{\prime} \\
\mathbf{S L S}_{3}{ }^{\prime}
\end{array}\right]\left[\begin{array}{r}
\Delta \mathbf{b}
\end{array}\right]
$$

where $\mathbf{S L S}_{1}{ }^{\prime}{ }_{1 i}=\gamma_{i} \mathbf{S L S}_{1,{ }_{1 i}}+\mathbf{S L S}_{2},,_{i} ; \mathbf{S L S}_{2}{ }^{\prime}{ }_{{ }_{1 i}}=\gamma_{i} \mathbf{S L S}_{3,{ }_{1 i}}+\mathbf{S L S}_{4},_{1 i}$;

$$
\begin{aligned}
& \gamma_{i}=\frac{\Delta g_{i}}{\Delta b_{i}}=\frac{\operatorname{Re}\left(\Delta g_{i}+j \Delta b_{i}\right)}{\operatorname{Im}\left(\Delta g_{i}+j \Delta b_{i}\right)}=\frac{\operatorname{Re}\left(\frac{1}{\left(1-\frac{\alpha_{i}}{100}\right) r_{i}+j\left(1-\frac{\beta_{i}}{100}\right) x_{i}}-\frac{1}{r_{i}+j x_{i}}\right)}{\operatorname{Im}\left(\frac{1}{\left(1-\frac{\alpha_{i}}{100}\right) r_{i}+j\left(1-\frac{\beta_{i}}{100}\right) x_{i}}-\frac{1}{r_{i}+j x_{i}}\right)} \\
& =\frac{\frac{\left(1-\frac{\alpha_{i}}{100}\right) r_{i}}{\left(1-\frac{\alpha_{i}}{100}\right)^{2} r_{i}^{2}+\left(1-\frac{\beta_{i}}{100}\right)^{2} x_{i}^{2}}-\frac{r_{i}}{r_{i}^{2}+x_{i}^{2}}}{\frac{-\left(1-\frac{\beta_{i}}{100}\right) x_{i}}{\left(1-\frac{\alpha_{i}}{100}\right)^{2} r_{i}^{2}+\left(1-\frac{\beta_{i}}{100}\right)^{2} x_{i}^{2}}-\frac{-x_{i}}{r_{i}^{2}+x_{i}^{2}}}=\frac{\left(1-\frac{\alpha_{i}}{100}\right) r_{i}\left(r_{i}^{2}+x_{i}^{2}\right)-r_{i}\left\{\left(1-\frac{\alpha_{i}}{100}\right)^{2} r_{i}^{2}+\left(1-\frac{\beta_{i}}{100}\right)^{2} x_{i}^{2}\right\}}{-\left(1-\frac{\beta_{i}}{100}\right) x_{i}\left(r_{i}^{2}+x_{i}^{2}\right)+x_{i}\left\{\left(1-\frac{\alpha_{i}}{100}\right)^{2} r_{i}^{2}+\left(1-\frac{\beta_{i}}{100}\right)^{2} x_{i}^{2}\right\}}(14)
\end{aligned}
$$

where $r_{i}$ is resistance of line $i[\mathrm{pu}], x_{i}$ is reactance of line $i[\mathrm{pu}]$.

Therefore, power loss changes are shown by the following equation:

$$
\left[\begin{array}{c}
\sum \Delta \text { Ploss }_{i} \\
\sum \Delta \text { Qloss }_{i}
\end{array}\right]=\left[\begin{array}{l}
\mathbf{S L S}_{1}{ }^{\prime} \\
\mathbf{S L S}_{3}{ }^{\prime}
\end{array}\right]\left[\begin{array}{l}
\mathbf{\Delta b}
\end{array}\right]
$$

Furthermore, the factors of $\Delta \mathbf{b}$ are not same because of the difference of the line distance, $\alpha_{i}$ and $\beta_{i}$. In other words, the rankings may change by multiplying $\Delta b_{i}$ respectively when we prioritize SLS'. Then, combining $\Delta b_{i}$ into SLS', we can transform SLS' to ESLC which is estimated system loss change as follows:

$$
\left[\begin{array}{l}
\Delta \text { Ploss } \\
\Delta \text { Qloss }
\end{array}\right]=\left[\begin{array}{l}
\text { SLS }_{1}{ }^{\prime} \\
\text { SLS }_{3}{ }^{\prime}
\end{array}\right][\Delta \mathbf{b}]=\left[\begin{array}{l}
\text { ESLC }_{1} \\
\text { ESLC }_{3}
\end{array}\right]\left[\begin{array}{c}
1 \\
\mathrm{M} \\
1
\end{array}\right]
$$

where $\mathbf{E S L C}_{1,{ }_{1 i}}=\Delta b_{i} \mathbf{S L S}_{1}{ }^{\prime}{ }_{1 i} ; \mathbf{E S L C}_{3,{ }_{1 i}}=\Delta b_{i} \mathbf{S L S}_{3}{ }^{\prime}{ }_{\jmath_{1 i}}$.

The factors of the column vector which is multiplied to ESLC are the same. We can accurately prioritize the transmission lines by the sensitivities.

\subsection{SLS Accuracy Confirmation}

We confirmed the accuracy of the SLS by using the IEEJ EAST 10 machine 47 bus system model $^{[2]}$. In the confirmation, we assumed $\alpha_{i}, \beta_{i}=-10$ [\%] and evaluated the whole system loss changes by a line renewal. The whole system loss changes are shown in Fig. 1 and Fig. 2 when each line was renewed. 

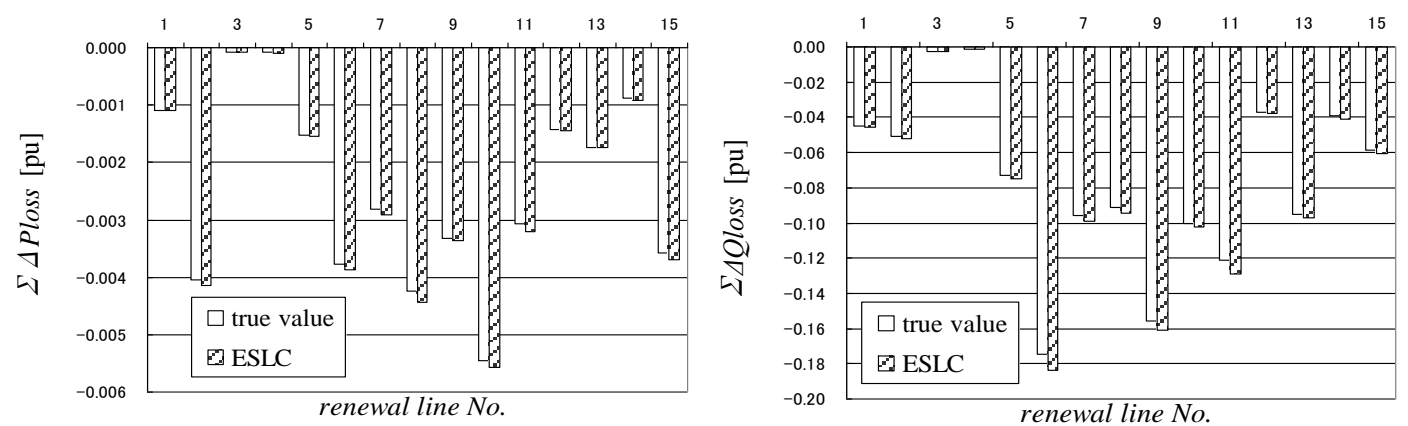

Fig. 1. Active power loss change by each line renewal.

Fig. 2. Reactive power loss change by each line renewal.

Judging from Fig. 1 and Fig. 2, we can confirm that there is little difference between the ESLC values and the true ones, and that the rankings of the lines are the same. In this paper, we prioritized ESLC $_{\mathbf{1}}$ because we aim to maximally decrease active power loss.

\subsection{Planning Method with the ESLC}

In this paper, we propose a new transmission line renewal planning method using the ESLC. We need to consider various factors in the actual planning, which are the ages of facilities, leveling of renewal cost, the terms of construction, and so on. However, we plan the line renewals which have high ESLC in order to confirm the validity of the ESLC. The flowchart of the proposed method is shown in Fig. 3.

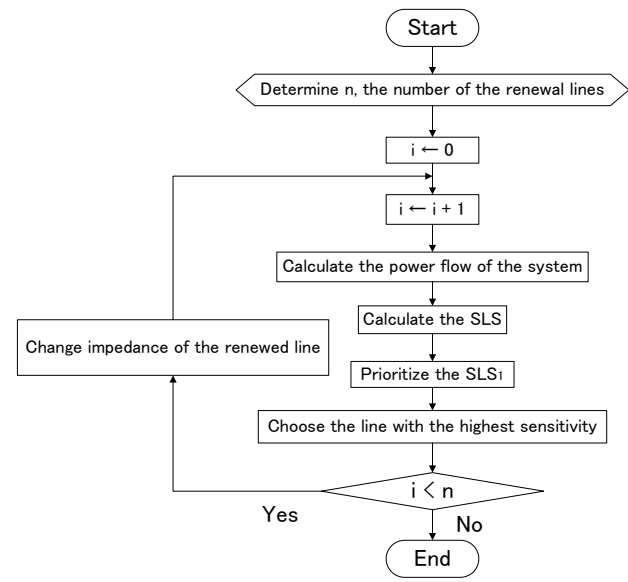

Fig. 3. Flowchart of the proposed method.

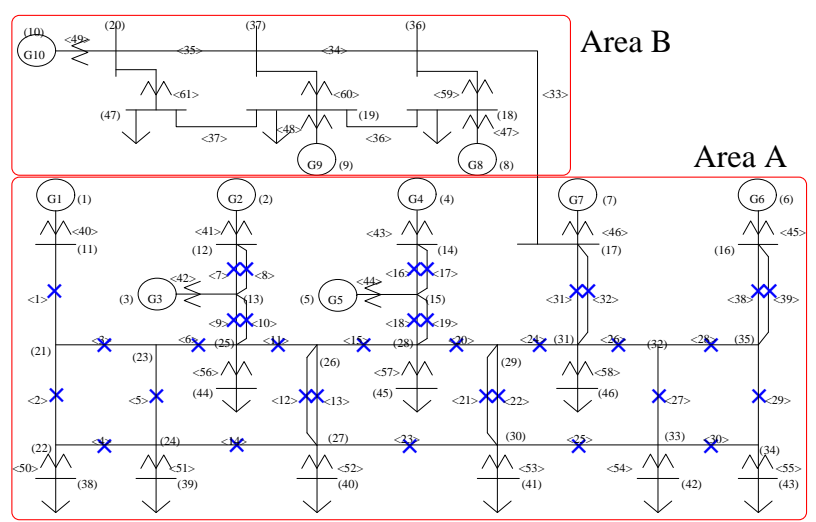

Fig. 4. IEEJ EAST 10 machine 47 bus system model [2].

\section{Simulations}

In this paper, we carried out the two simulations below using the IEEJ EAST 10 machine 47 bus system model [2] to confirm the validity of the proposed method. The system is shown in Fig. 4. Here, the kind of the transmissions used in the simulation model was "TACSR810 [mm $\left.{ }^{2}\right] 4$ conductors," and the impedance of one line per the length of 100 [km] was as follows [4].

$$
Z=r+j x=0.0046+j 0.1068 \text { [p.u.] }
$$

- Simulation 1: Compare high ranking lines and others

We compare the line renewals whose ESLC rankings were 1st-10th with those whose rankings were 11th-20th by evaluating the amount of the loss improvements and the bus voltage improvements.

- Simulation 2: Assume N-1 contingency

We confirm the selecting method in the original system was valid in the $\mathrm{N}-1$ contingency system. 


\subsection{Assumptions for simulations}

In this paper, we carried out simulations based on the below assumptions:

- The impedances of the renewed lines are changed to 90-99 [\%] of their original values. $\left(\alpha_{i}, \beta_{i}=\right.$ $-10--1[\%])$.

- The renewal candidates are the lines shown as crossed in Area A.

- We evaluate the influence of 10 line renewals.

\subsection{Results of simulation 1}

The amount of the loss improvements are shown in Table 1 and Table 2, which are calculated after renewing the 10 highest ESLC ranking (1st-10th) lines and the second 10 highest ESLC ranking lines (11th-20th), respectively. Here, we did not consider the voltages of the bus in Area B.

Judging from Table 1 and Table 2, the ESLC high ranking line renewals have more influence concerning the loss improvements.

Thus, we confirmed the validity of the proposed method for the original system.

Table 1. System active power loss change

\begin{tabular}{|c|c|c|}
\hline & ranking $1-10$ & ranking $11-20$ \\
\hline EPloss (before) [pu] & 1.432 & 1.432 \\
\hline EPloss (after) [pu] & 1.365 & 1.399 \\
\hline$\Delta(\Sigma$ Ploss $)$ & -0.067 & -0.033 \\
\hline percentage & 95.33 & 97.68 \\
\hline
\end{tabular}

Table 2. System reactive power loss change

\begin{tabular}{|c|c|c|}
\hline & ranking $1-10$ & ranking $11-20$ \\
\hline EQloss (before) [pu] & 45.095 & 45.095 \\
\hline$\sum$ Qloss (after) [pu] & 43.384 & 44.232 \\
\hline$\Delta(\Sigma$ Qloss $)$ & -1.711 & -0.863 \\
\hline percentage & 96.21 & 98.09 \\
\hline
\end{tabular}

\subsection{Results of Simulation 2}

Simulations were run to confirm the validity of the 10 highest ESLC ranking line renewals in an N-1 contingency, which was chosen in Simulation 1. The amount of loss improvements achieved by the ESLC ranking from 1 to 10 line renewals is shown in Table 3, and the bus voltages in Fig. 5 . The N-1 contingency is of line No.1 which gives the most severe fault case (Opening one line out of a double circuit line).

Table 3. System loss change of the N-1 contingency system

\begin{tabular}{|c|c|}
\hline & ranking $1-10$ \\
\hline EPloss (before) [pu] & 1.705 \\
\hline EPloss (after) [pu] & 1.586 \\
\hline$\Delta$ (sPloss $)$ & -0.119 \\
\hline percentage & 93.02 \\
\hline
\end{tabular}

\begin{tabular}{|c|c|}
\hline & ranking $1-10$ \\
\hline$\Sigma$ Qloss (before) [pu] & 53.832 \\
\hline$\Sigma$ Qloss (after) [pu] & 50.357 \\
\hline 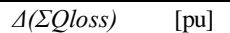 & -3.476 \\
\hline percentage & 93.54 \\
\hline
\end{tabular}

Table 4. SLS rankings of the initial system and the N-1 contingency system

\begin{tabular}{|c|c|c|}
\hline ranking & initial system & N-1 contingency(Line No.1) \\
\hline 1 & 31 & 31 \\
\hline 2 & 29 & 6 \\
\hline 3 & 19 & 38 \\
\hline 4 & 27 \\
\hline 5 & 38 & 27 \\
\hline 6 & 24 \\
\hline 7 & 10 & 19 \\
\hline 8 & 8 & 24 \\
\hline 9 & 2 & 29 \\
\hline 10 & 22 & 10 \\
\hline
\end{tabular}

We confirmed greater improvement in the N-1 contingency from Fig. 5, compared with that of the original system. We believe the reason is that the N-1 contingency system has a bigger reactive power loss of $53.832[\mathrm{pu}]$ compared with the value of 50.357 [pu] of the original system, and the amount of the reactive power loss improvement is changed to $3.476[\mathrm{pu}]$ from $1.711[\mathrm{pu}]$ which is smaller by more than $50[\%]$.

Furthermore, the agreement of the ESLC rankings of the N-1 contingency system and the original one is considered another reason why the bus voltages greatly improve (Shown in Table 4). 
From Table 4, we confirmed there is little change between the 10 highest ranking lines of the N-1 contingency system and the original one.

Also, the bus voltage drops by the $\mathrm{N}-1$ contingency are improved more than the other buses.

Thus, we confirmed the validity of the proposed method for the N-1 contingency case as well.

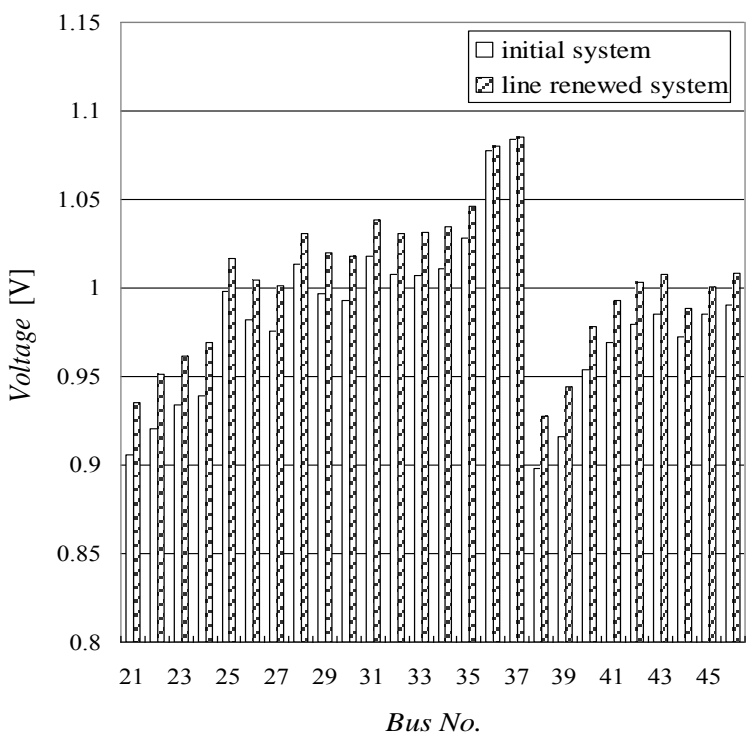

Fig. 5. Voltages of the load buses in the N-1 contingency system.

\section{Conclusion}

In this paper we proposed a new transmission renewal planning index, "ESLC," considering both the decrease of active power flow loss and the voltage quality improvement due to the decrease of reactive power flow loss. In the simulations, by implementing the line renewals determined by the ESLC, we first confirmed the active power loss improvement in the original system, and next, by implementing the above renewals for the N-1 contingency case, we also confirmed the efficient bus voltage improvements. Therefore, it was confirmed that the proposed index is very beneficial in prioritizing candidate lines for transmission renewal planning.

\section{References}

[1] Tanaka H, Takehara A, Shibata T Nagata M. Proposal of maintenance planning support method for transmission facility based on supply reliability and cost evaluation - proposal of a life-cycle risk map for maintenance planning. Research report. System Engineering Research Laboratory Rep., No. R07030, July 2008 (in Japanese).

[2] Grainger J. Stevenson W. Power System Analysis, McGraw-Hill, New York; 1994

[3] The Technical Committee of the Institute of Electrical Engineers in Japan (IEEJ). Japanese power standard system models. Technical report. IEEJ Technical Report, 754:10-15, 1999 (in Japanese).

[4] Dong ZY, Lu M, Lu Z, Wong KP. A differential evolution based method for power system planning. Presented at: IEEE Congress on Evolutionary Computation, 2006.

[5] Ng WY. Generalized generation distribution factors for power system security evaluation. Transaction on Power Apparatus and Systems, 1981; 100(3):1001-1005.

[6] Bialek J. Tracing the flow of electricity. IEE Proceeding on Generation, Transmission and Distribution, 1996; 143(3):313-320.

[7] M Pantos, Verbic G, Gubina F. Modified topological generation and load distribution factors. IEEE Transactions on Power Systems, 2005; 20(4):1998-2005.

[8] Jibiki T, Sakakibara E, Iwamoto S. Line flow sensitivities of line reactances for congestion management. Presented at: IEEE PES General Meeting, 2007. 